\title{
The Strategy Tool: The Trademark Map of Best 100 Brands in the World
}

\author{
Rain Chen ${ }^{1} \&$ Chapie Liang ${ }^{2}$ \\ ${ }^{1}$ Department of Creative Product Design, Southern Taiwan University of Science and Technology (STUST), Tainan, \\ Taiwan, R.O.C. \\ ${ }^{2}$ Graduate Institute of Digital Content and Animation Design, Southern Taiwan University of Science and \\ Technology (STUST), Tainan, Taiwan, R.O.C. \\ Correspondence: Dr. Rain Chen, Department of Creative Product Design, Southern Taiwan University of Science \\ and Technology (STUST), No. 1, Nan-Tai Street, Yongkang Dist., Tainan City 710, Taiwan, R.O.C. Tel: \\ 886-6-253-3131 ext. 8107, 886-6-301-0020. E-mail: ocean@mail.stust.edu.tw
}

Received: October 1, 2013

Accepted:October 14, 2013

Online Published: November 8, 2013

doi:10.5430/jms.v4n4p21

URL: http://dx.doi.org/10.5430/jms.v4n4p21

\begin{abstract}
In this research, five steps are brought up to build up the trademark map, including (1) deciding sample range of trademarks, (2) analyzing the first-time information, (3) analyzing the second-time information, (4) building up the trademark map, and (5) analyzing the trademark map. This standard procedure can help enterprises create their trademark maps efficiently. A multi-dimensional scale is used for analyzing and building up the trademark map of the most famous one hundred brands, and 86 consumers are requested to proceed with the experiment of brand identification. The results are shown as follows. (1) To display the distribution of trademark samples clearly by building a visualized map, the level of trademark similarity between samples can be understood. (2) Enterprises can the apply trademark map for judging the identification and feasibility of their trademarks so that they are capable of avoiding tort and creating their own and only brand image.
\end{abstract}

Keywords: brand management, trademark map, consumer identification, brand position, brand strategy

\section{Introduction}

The brand image and self-image of consumers can be the crutial causes of purchase behavior. The more famous the brand is shown, the phenomenon can be more obvious. Therefore, illegal businessmen tend to interfere consumers' identification with similar trademarks causing confusion and misjudgement and then lead to damaging consumers' rights (Mealema etc, 2010). Trademark has been taken as a central core in an enterprise. For enterprises, relating their images to some rough products in the market by consumers can be a serious problem of degrading their trademark value (Suzuki, 2002).

Brand is not only a logo of products, but also a main reason for influencing purchase behavior (Berry etc., 2007). The moment of consumers reaching their hands for a familiar, safe, trusty product, brand will be a crucial factor (Delgado-Ballester etc., 2005). People are willing to offer more money for similar but much more famous products (Kort etc., 2006; Berthon etc., 2009); therefore, most of the corporations try to promote their products and services by brand expansion.

In the time of a brand which is famous enough for consumers creating associating thinking, brand loyalty starts to be established (Olavarrieta etc., 2009). However, it takes time to accumulate brand reputation (Veloutsou etc., 2009), and brand faith cannot be created over one night as well. Consumers need lots of time to understand the spirit of a brand (Delgado-Ballester etc., 2001). Therefore, creating brand reputation and image has become the crucial goal of brand management (Torres-Moraga etc., 2008). Due to the level of brand satisfaction being able to affect the next purchase of a consumer (Ewing, 2000), service quality and credit of a brand become an important factor (Rauyruen etc., 2009). In addition, finding a way for costumers establishing brand loyalty will raise the ratio for consumers buying the product again (Huber etc., 2009; Shukla, 2009).

After purchasing a product (or a service) through brand identification, brand has delivered the value of its product (or service) to consumers, and a consumer can gain invisible emotion besides the actual product (or service). At the time consumers accumulating their positive view about a brand, the brand value is accomplished. Brand value is an invisible property of a corporation. Continually increasing value can make a brand much more well-known and 
become a strong support of the company (Gabay etc., 2009). However, there is no visual map of trademarks related research.

Enterprises take trademark as a praised product or a combination with logo, symbol, and mark of its service brand, which is also used for separating other similar products in the market (Palumbo etc., 2000). The benefits of having a trademark contain assisting consumer in identifying the product, understanding the source and quality of the product, and making enterprises well-known in public (Barnes, 2009). Trademarks are positively being taken charge of product sales and enterprise development. WIPO (World Intellectual Property Organization) has defined trademark widely as "making trademark owner gain brand awarenss and profit, and preventing selling product from competitors and their similar trademark or service". Therefore, trademark protection has become a common consensus in many countries, which start to legislate for relevant issues.

Due to the main principle of trademark law being to become the first resgister, trademark must be protected at the beginning of application, for example, resgisteration. Before registering a trademark, one has to search the current database for any similar trademarks to avoid tort (Qi etc., 2010). After registration, the trademark owner should start paying attention carefully to similar or any other trademarks which can lead to confusion and make sure the legal right for their trademark not being harmed. Substantive scope of trademark rights so far not been objectively defined.

The key function of trademark is for consumer indentifying the brand of products or services. However, two different trademarks with the combination of some similar parts indicate harassment. The types of trademark similarity mostly are apperence similarity pronunciation similarity, and concept similarity.

To judge the similarity of two trademarks, it depends on objective consumers with common sense. When there is confusion and mix-up, these two trademarks are similar. The main principles to judge trademark similarity are (1) fully observation, (2) observing at different time and different places, and (3) the level of similarity within the entire image. In fact, there exists no specific procedure and solution to judge trademark similarity.

The trademark mix-up is a phenomenon about consumers misjudging the source of products or services due to the trademark similarity. According to the WIPO, the examination standards are published and eight judging factors are brought up (TIPO, 2012) as (1) level strength of distinctiveness of the trademark(s), (2) whether the trademarks are similar and if yes, the extent of similarity between them, (3) whether the goods/services are similar and if yes, the extent of similarity between them, (4) status of the diversified operation of the prior right holder, (5) circumstances of actual confusion, (6) the extent to which relevant consumers are familiar with the trademarks concerned, (7) whether the applicant of the trademark at issue in question has filed such application in good faith, and (8) other factors that may cause confusion.

The judgment of trademark mix-up is mainly to clarify whether the trademark confusing costumer or not, and to make sure whether the applicant of trademark cause any violation against other peoples' legal rights or not. Even though there are strict law for trademark protection to assist people in judging trademark mix-up and confusion, trademark designers and owners will not enjoy being prohibited using their own trademark due to similarity problem, and they even need to go to court. Therefore, the study of logo similarity does have extraodinary impact on enterprises.

Due to trademark being a symbol of a brand, businessmen can have the monopoly to use it for the company or products (George, 2006). Therefore, when there are a relative thought between two brands, there possibly exists tort (Arvidsson, 2006). Most of tort happened in mid-small enterprises or a person commited. Some of them are intentionally acts, and the others are unpremeditated torts. The proportion of unpremeditated tort is high. Besides, the major reason of tort particially is from unclear definition and standards in logo similarity. In addition, in the real case, judges tend to compare the complained trademark and defended trademark alone, and make every trademark tort become a single case. The result of a single case can not be applied to the tort trial. Therefore, how to use an objective way to build up a full-scale trademark map for judges, enterprises, logo designers and consumers to judge trademark similarity has become an important issue.

In the time of enterprises pursuing global brand distribution becomes a trend, the production of trademark map and search engine toward trademarks becomes more important as well. Enterprises need to be familiar with competitors' trademark distribution before promoting their brand to the world. In this research, the way of building patent map is taken as a reference to create enterprise brand into a trademark map. This research can display the level of trademark similarity clearly and ensure the feasibility of trademark application.

Through building up a trademark map to visualize trademark orientation, so far the research on trademark map is limited, but not this research! This research can be applied to the fields of (1) creating an objective view about 
trademarks for judges judging tort by building up the related trademark map, (2) enterprises beign able to build up a trademark map within its field to find its position in the market, and (3) trademark designers bein able to build up trademark maps of their concepts, and use it for avoiding tort while designing trademarks.

\section{Method}

Every intellectual property organization in advanced countries, like United States Patent and Trademark Office (USPTO), European Patent Office (EPO), and Japan Patent Office (JPO) etc., can be divided the published references about trademarks into two categories. (1) Background describing data (it can also be called one-time data). Like trademark serial number, application data of trademarks, publishing day of registration...etc, these describe information about trademark background. (2) Technical context data (also be known as second-time data). It means the main body of the trademark design and the symbol of the brand. The second-time data are more subjective, but have more values in it. Building up a trademark map is an analysis and criticition toward both the one-time and the second-time data.

\subsection{Subjects}

The main purpose of trademarks is to avoid confusion and mix-up problems of consumers. In addition, there are three principles to judge trademark similarity, including (1) full observation, (2) observing at different time and different places, and (3) the level of similarity within the entire image and all ordinary customers being the main subjects. Therefore, normal consumers are regarded as the testing subjects to observe where about trademarks consumers with common purchase experience can be confused while paying attention to products. Due to the sharper observation from trademark designers and people related to trademark firms, trademark designers and related people are deliberately eliminated, and only normal customers are the main subjects. Besides, sampling with simples and random principles are utilized.

\subsection{Brand Samples}

The estimating report with credibility about the best 100 brands in the world was published by Brandz (2009) and Interbrand (2010). Due to different pricing ways, the results were various. Brandz followed 650,000 consumers and experts fixedly, and Interbrand emphasized the expert research. The major function of trademarks is providing product identification to increase the chance of repeatly purchase that the point of view from consumer is more important. Therefore, the published brand report from Brandz was used as the proinciple resource of studying samples. In addition, to avoid people tending to focus on the most familiar trademarks, the brand report within this year were skipped, and the published report of the best 100 brands in the world was taken as the major studying samples. The selecting samples are as follows in Table 1.

Table 1. The best 100 brands in 2009 (Data source: Brandz 2009 Report)

\begin{tabular}{|c|c|c|c|c|c|c|c|c|c|}
\hline No. & Brand Name & No. & Brand Name & No. & Brand Name & No. & Brand Name & No. & Brand Name \\
\hline 1 & Google & 21 & TESCO & 41 & $\begin{array}{l}\text { Bank of } \\
\text { america }\end{array}$ & 61 & TD & 81 & Yahoo \\
\hline 2 & Microsoft & 22 & Gillette & 42 & Dell & 62 & Movistar & 82 & Hermes \\
\hline 3 & Cocacola & 23 & Intel & 43 & Accenture & 63 & T mobile & 83 & J.P.morgan \\
\hline 4 & IBM & 24 & $\begin{array}{l}\text { China } \\
\text { construction } \\
\text { bank }\end{array}$ & 44 & Pepsi & 64 & Wrigleys & 84 & Ariel \\
\hline 5 & Mcdonals & 25 & Oracle & 45 & Loreal & 65 & Auchan & 85 & Tide \\
\hline 6 & Apple & 26 & Amazon & 46 & $\begin{array}{l}\text { American } \\
\text { Express }\end{array}$ & 66 & Chase & 86 & Gucci \\
\hline 7 & $\begin{array}{l}\text { China } \\
\text { mobile }\end{array}$ & 27 & $\begin{array}{l}\text { Bank of } \\
\text { china }\end{array}$ & 47 & Carrefour & 67 & Nissan & 87 & MasterCard \\
\hline 8 & GE & 28 & At\&t & 48 & $\mathrm{RBC}$ & 68 & DHL & 88 & $\begin{array}{l}\text { Goldman } \\
\text { sachs }\end{array}$ \\
\hline 9 & Vodafone & 29 & LV & 49 & Citi & 69 & Fedex & 89 & Starbucks \\
\hline 10 & Marlboro & 30 & HSBC & 50 & Honda & 70 & $\begin{array}{l}\text { Home } \\
\text { depot }\end{array}$ & 90 & Barclays \\
\hline 11 & Walmart & 31 & Pampers & 51 & Siemens & 71 & MTS & 91 & State farm \\
\hline
\end{tabular}




\begin{tabular}{llllllllll}
\hline 12 & ICBC & 32 & Nintendo & 52 & Budweiser & 72 & Eunauh & 92 & $\begin{array}{l}\text { Morgan } \\
\text { Stanley }\end{array}$ \\
13 & Nokia & 33 & Cisco & 53 & Orange & 73 & Canon & 93 & ING \\
14 & Toyota & 34 & Verizon & 54 & Ebay & 74 & ALDI & 94 & KFC \\
15 & UPS & 35 & Porsche & 55 & BBVA & 75 & AVON & 95 & IKEA \\
16 & Blackberry & 36 & VISA & 56 & Colgate & 76 & Zara & 96 & NIVEA \\
17 & HP & 37 & Wells fargo & 57 & Target & 77 & O2 & 97 & Esprit \\
18 & BMW & 38 & Santander & 58 & H\&M & 78 & Standard & 98 & Bradesco \\
& SAP & 39 & Docomo & 59 & Nike & 79 & $\begin{array}{l}\text { Red bull } \\
\text { china }\end{array}$ & 99 & TIM \\
20 & Disney & 40 & Benz & 60 & Subway & 80 & Lowes \\
& & & & & & & merchants & & \\
\hline
\end{tabular}

\subsection{Experimental Design}

To move forward and transfer one-time data into second-time data, authors made the trademark samples of the famous 100 brands into graphic cards, and the cards only showed black trademark and sample number (as in Figure 1). Besides the graphic card, any related information was not provided in case of unnecessary interference. Then, 100 normal consumers were requested to devide the trademark samples into groups according to their similarityies. The grouping results were screened by examining the questions in questionnaires that 14 questionnaires were eliminated for 86 efficient copies.

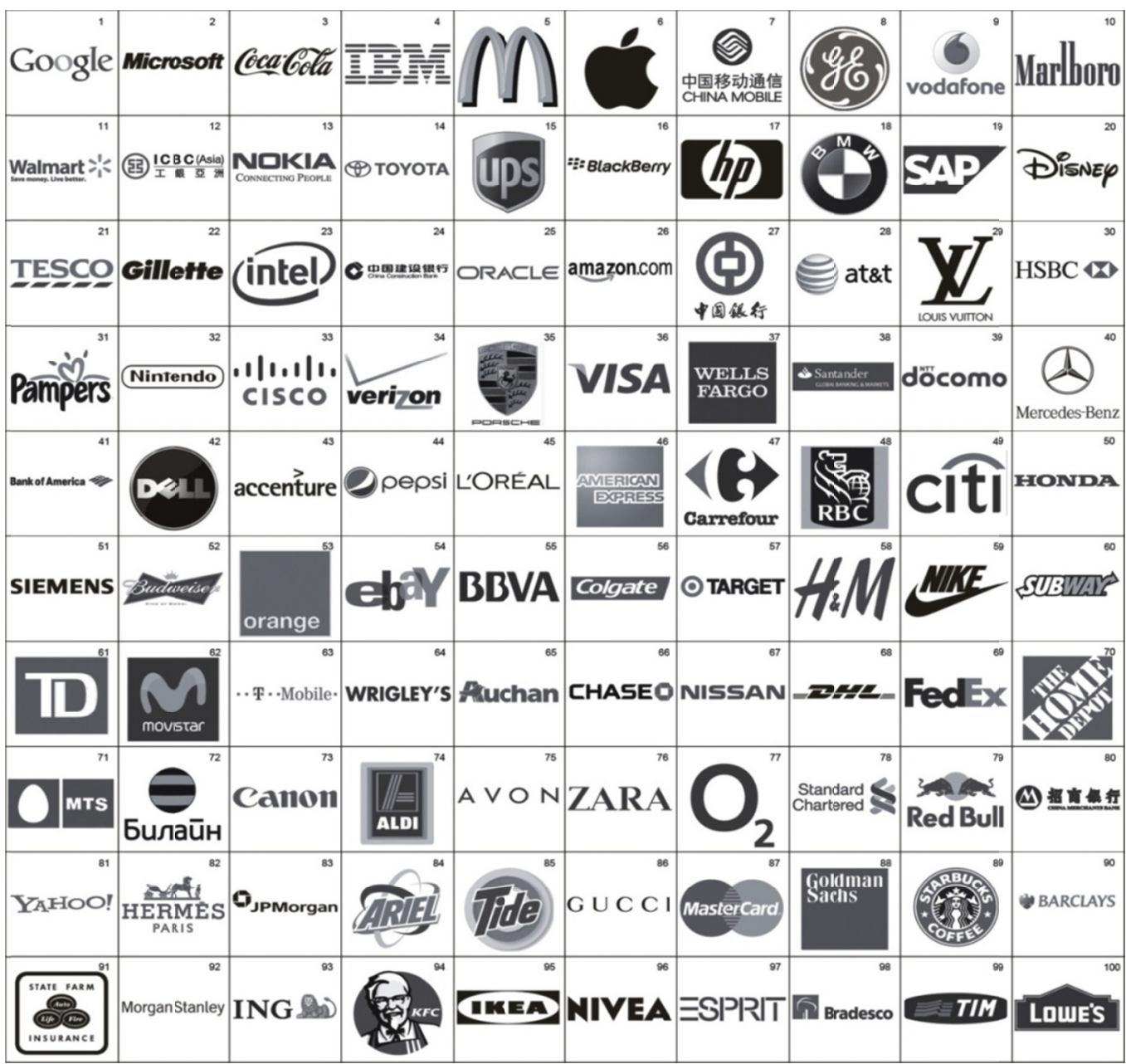

Figure 1. The trademark samples of the best 100 brands in the world 
After the consumers dividing the graphic cards, the authors moved forward to organize the grouping results. Appendix 1 shows the result of grouping by normal consumers. Taking Appendix 1 as the explaining sample, the number in the matrix of trademark samples number 3 and 5 were 67, indicating that 67 out of 86 consumers considered that the samples number 3 and 5 were not similar (the rest 19 consumers believed that these two samples were similar). Janssensa once applied MDS in information science (Janssensa, 2006) and achieved the demand for visualizing the trademark information. Therefore, multi-dimensional scale (MDS) was used for analyzing Appendix 1.

\section{Result}

Total 100 questionnaires were sent out to normal consumers. Having eliminated 14 invalid questionnaires, there were 86 effective questionnaires. The following information is the basic descriptions from the 86 effective questionnaires. (1) Gender: 39 male subjects (45.3\%), 47 female subjects (54.7\%), and the ratio of men and women was 1 to 1.2.(2)Age: There were 68 people aged between 21 to 30, and the ratio was $79.1 \%, 12$ people between 31 to 40 years old (14\%), 3 people between 51 to 60 years old (3.5\%), 2 people under 20 years old $(2.3 \%)$, and only one person between 41-50 years old. (3) Education: There were 67 college students (77.9\%), 15 graduate students (17.4\%), 2 high school students $(2.3 \%)$ and 2 junior high students $(2.3 \%)$.

There are five steps to create a trademark map, as (1) setting the trademark sample range, (2) analyzing one-time data, (3) analyzing second-time data, (4) building up trademark map, and (5) analyzing trademark map. These five steps will be described separately as follows.

\subsection{Setting Range of Trademark Samples}

According to the trademark newspaper published by Intellectual Property Office in Taiwan, six crucial descriptions of trademark background were extracted, including (1) Registration Number, (2) Filing Date, (3) Registration Date, (4) Nationality, (5) Owner, and (6) Brand Name. The background descriptions from trademark newspaper within the best 100 brands in the world are listed in Table 2.

Table 2. The background description from trademark newspaper within the best 100 brands in the world

\begin{tabular}{|c|c|c|c|c|c|c|}
\hline No. & $\begin{array}{l}\text { Registratio } \\
\text { n Number }\end{array}$ & Filing Date & $\begin{array}{l}\text { Registratio } \\
\text { n Date }\end{array}$ & Nationality & Owner & Brand Name \\
\hline 1 & 4123471 & $2004 / 6 / 10$ & $2012 / 4 / 10$ & United States & Google, Inc. & Google \\
\hline 2 & 1087879 & $2010 / 11 / 2$ & $2011 / 5 / 24$ & United States & Microsoft Corporation & Microsoft \\
\hline 3 & 1277043 & $1982 / 2 / 3$ & $1984 / 5 / 8$ & United States & The Coca-Cola Company & Cocacola \\
\hline 4 & 1205090 & $1980 / 4 / 30$ & $1982 / 8 / 17$ & United States & $\begin{array}{l}\text { International Business } \\
\text { Machines Corporation }\end{array}$ & IBM \\
\hline 5 & 2393485 & $1999 / 10 / 26$ & $2000 / 10 / 10$ & United States & Mcdonald's Corporation & Mcdonals \\
\hline 6 & 2180949 & $1995 / 8 / 8$ & 1998/8/11 & United States & Apple Computer, Inc. & Apple \\
\hline 7 & 2878429 & $2002 / 10 / 28$ & $2004 / 8 / 31$ & China & China Mobile & China Mobile \\
\hline 8 & 0878049 & $1969 / 3 / 14$ & $1969 / 10 / 7$ & United States & General Electric Company & $\mathrm{Ge}$ \\
\hline 9 & 3561152 & $2006 / 3 / 17$ & $2009 / 1 / 13$ & Kingdom & Vodafone Group Plc & Vodafone \\
\hline 10 & 0068502 & $1907 / 10 / 17$ & $1908 / 4 / 14$ & United States & Philip Morris Brands Sarl & Marlboro \\
\hline 11 & 3546870 & $2008 / 7 / 1$ & $2008 / 12 / 16$ & United States & Wal-Mart Stores, Inc. & Walmart \\
\hline 12 & 2921910 & $2001 / 6 / 5$ & $2005 / 2 / 1$ & United States & Icbc Bank & ICBC \\
\hline 13 & 0871846 & $2005 / 11 / 18$ & $2009 / 6 / 23$ & $\begin{array}{l}\text { Northern } \\
\text { Europe }\end{array}$ & Nokia Corporation & Nokia \\
\hline 14 & 3655725 & $2008 / 6 / 30$ & $2009 / 7 / 14$ & Japan & Toyota Group & Toyota \\
\hline 15 & 2867999 & $2003 / 3 / 24$ & $2004 / 7 / 27$ & United States & $\begin{array}{l}\text { United Parcel Service Of } \\
\text { America, Inc. }\end{array}$ & Ups \\
\hline 16 & 3102687 & $2004 / 9 / 3$ & $2006 / 6 / 13$ & Canada & Research In Motion Limited & Blackberry \\
\hline 17 & 1251648 & $1982 / 9 / 13$ & $1983 / 9 / 20$ & United States & Hewlett-Packard Company & Нp \\
\hline 18 & 1450212 & $1984 / 6 / 29$ & $1987 / 8 / 4$ & Germany & $\begin{array}{l}\text { Bayerische Motoren Werke } \\
\text { Aktiengesellschaft }\end{array}$ & $\mathrm{Bmw}$ \\
\hline 19 & 2688100 & $2001 / 8 / 1$ & $2003 / 2 / 18$ & Germany & Sap Aktiengesellschaft & Sap \\
\hline 20 & 3548154 & $2007 / 4 / 5$ & $2008 / 12 / 16$ & United States & Disney Enterprises, Inc. & Disney \\
\hline 21 & 3195448 & $2004 / 11 / 4$ & $2007 / 1 / 9$ & Canada & Tesco Stores Limited & Tesco \\
\hline 22 & 3547795 & $2007 / 8 / 27$ & $2008 / 12 / 16$ & United States & The Gillette Company & Gillette \\
\hline
\end{tabular}




\begin{tabular}{|c|c|c|c|c|c|c|}
\hline 23 & 3730085 & $2005 / 12 / 21$ & $2009 / 12 / 22$ & United States & Intel Corporation & Intel \\
\hline 24 & 3098324 & $2004 / 5 / 21$ & $2006 / 5 / 30$ & China & China Construction Bank & $\begin{array}{l}\text { China } \\
\text { Construction } \\
\text { Bank }\end{array}$ \\
\hline 25 & 3619756 & $2006 / 10 / 17$ & $2009 / 5 / 12$ & United States & Oracle Corporation & Oracle \\
\hline 26 & 2789101 & $2000 / 1 / 12$ & $2003 / 12 / 2$ & United States & Amazon Technologies, Inc. & Amazon \\
\hline 27 & 3182253 & $2005 / 4 / 4$ & $2006 / 12 / 12$ & China & Bank Of China & $\begin{array}{l}\text { Bank Of } \\
\text { China }\end{array}$ \\
\hline 28 & 1688845 & $1991 / 4 / 26$ & $1992 / 5 / 26$ & United States & At\&T Corp. & At\&T \\
\hline 29 & 1519828 & $1988 / 5 / 6$ & $1989 / 1 / 10$ & France & Vuitton \& Fils & LV \\
\hline 30 & 2000657 & $1994 / 10 / 18$ & $1996 / 9 / 17$ & $\begin{array}{l}\text { United } \\
\text { Kingdom }\end{array}$ & $\begin{array}{l}\text { The Hongkong And Shanghai } \\
\text { Banking Corporation }\end{array}$ & Hsbc \\
\hline 31 & 2821456 & $2000 / 3 / 6$ & $2004 / 3 / 9$ & United States & $\begin{array}{l}\text { The Procter \& Gamble } \\
\text { Company }\end{array}$ & Pampers \\
\hline 32 & 1689015 & $1991 / 1 / 4$ & $1992 / 5 / 26$ & United States & Nintendo Co., Ltd. & Nintendo \\
\hline 33 & 3747597 & $2008 / 10 / 15$ & $2010 / 2 / 9$ & United States & Cisco Technology, Inc. & Cisco \\
\hline 34 & 2879802 & $2000 / 3 / 3$ & $2004 / 8 / 31$ & United States & $\begin{array}{l}\text { Verizon Trademark Services, } \\
\text { Llc. }\end{array}$ & Verizon \\
\hline 35 & 0991621 & $1972 / 3 / 10$ & $1974 / 8 / 20$ & Germany & $\begin{array}{l}\text { Dr. Ing. H.C. F. Porsche } \\
\text { Aktiengesellschaft }\end{array}$ & Porsche \\
\hline 36 & 3357216 & $2005 / 4 / 8$ & $2007 / 12 / 18$ & United States & $\begin{array}{l}\text { Isa International Service } \\
\text { Association }\end{array}$ & Visa \\
\hline 37 & 2526696 & $2000 / 1 / 5$ & $2002 / 1 / 8$ & United States & Wells Fargo \& Company & Wells Fargo \\
\hline 38 & 4120918 & $2011 / 6 / 2$ & $2012 / 4 / 3$ & Spain & $\begin{array}{l}\text { Santander Investment Bank, } \\
\text { Ltd. }\end{array}$ & Santander \\
\hline 39 & 3370301 & $2008 / 6 / 12$ & $2012 / 5 / 22$ & Japan & Ntt Docomo, Inc. & Docomo \\
\hline 40 & 0285557 & $1929 / 8 / 16$ & $1931 / 7 / 28$ & Germany & $\begin{array}{l}\text { Daimler-Benz } \\
\text { Aktiengesellschaft }\end{array}$ & Benz \\
\hline 41 & 4146809 & $2011 / 12 / 9$ & $2012 / 5 / 22$ & United States & Bank Of America Corporation & $\begin{array}{l}\text { Bank Of } \\
\text { America }\end{array}$ \\
\hline 42 & 1860272 & $1992 / 2 / 27$ & $1994 / 10 / 25$ & United States & Dell Computer Corporation & Dell \\
\hline 43 & 3862419 & $2008 / 6 / 18$ & $2010 / 10 / 19$ & Switzerland & $\begin{array}{l}\text { Accenture Global Services } \\
\text { Gmbh }\end{array}$ & Accenture \\
\hline 44 & 3684305 & $2008 / 10 / 6$ & $2009 / 9 / 15$ & United States & Pepsico, Inc. & Pepsi \\
\hline 45 & 0661746 & $1956 / 6 / 25$ & $1958 / 5 / 13$ & France & L'oreal & Loreal \\
\hline 46 & 1032516 & $1975 / 1 / 27$ & $1976 / 2 / 3$ & United States & Carreras Limited & $\begin{array}{l}\text { American } \\
\text { Express }\end{array}$ \\
\hline 47 & 3720625 & $2008 / 4 / 16$ & $2009 / 12 / 8$ & France & Carrefour & Carrefour \\
\hline 48 & 2885583 & $2001 / 8 / 10$ & $2004 / 9 / 21$ & Canada & Royal Bank Of Canada & $\mathrm{Rbc}$ \\
\hline 49 & 3441460 & $2006 / 12 / 7$ & $2008 / 6 / 3$ & United States & Citicorp & Citi \\
\hline 50 & 3108842 & $2004 / 12 / 9$ & $2006 / 6 / 27$ & Japan & Honda Group & Honda \\
\hline 51 & 2392496 & $1997 / 4 / 3$ & $2000 / 10 / 10$ & Germany & Siemens Aktiengesellschaft & Siemens \\
\hline 52 & 3715882 & 2007/10/16 & $2009 / 11 / 24$ & United States & Anheuser-Busch, Incorporated & Budweiser \\
\hline 53 & 3722405 & $2005 / 7 / 22$ & $2009 / 12 / 8$ & $\begin{array}{l}\text { United } \\
\text { Kingdom }\end{array}$ & $\begin{array}{l}\text { Orange International } \\
\text { Developments Ltd. }\end{array}$ & Orange \\
\hline 54 & 3380423 & $2007 / 2 / 9$ & $2008 / 2 / 12$ & United States & Ebay, Inc. & Ebay \\
\hline 55 & 2657695 & $2000 / 1 / 28$ & $2002 / 12 / 10$ & Spain & $\begin{array}{l}\text { Banco Bilbao Vizcaya } \\
\text { Argentaria,S.A. }\end{array}$ & Bbva \\
\hline 56 & 3450987 & $2005 / 10 / 25$ & $2008 / 6 / 17$ & United States & Colgate-Palmolive Company & Colgate \\
\hline 57 & 3229570 & $2006 / 6 / 1$ & $2007 / 4 / 17$ & United States & Target Brands, Inc. & Target \\
\hline 58 & 3992496 & $2010 / 8 / 10$ & 2011/7/12 & Sweden & $\mathrm{H} \& \mathrm{M}$ Hennes \& Mauritz $\mathrm{Ab}$ & $\mathrm{H} \& \mathrm{M}$ \\
\hline 59 & 1595356 & $1989 / 10 / 13$ & $1990 / 5 / 8$ & United States & Nike International Ltd. & Nike \\
\hline 60 & 3869196 & $2009 / 8 / 24$ & $2010 / 11 / 2$ & United States & Doctor's Associates, Inc. & Subway \\
\hline 61 & 3037995 & $2004 / 11 / 15$ & $2006 / 1 / 3$ & Canada & Td Bank & TD \\
\hline 62 & 3283182 & $2005 / 7 / 19$ & $2007 / 8 / 21$ & Spain & Telefonica, S.A. & Movistar \\
\hline
\end{tabular}




\begin{tabular}{|c|c|c|c|c|c|c|}
\hline 63 & 3662569 & $2008 / 4 / 18$ & $2009 / 8 / 4$ & Germany & Deutsche Telekom Ag & T Mobile \\
\hline 64 & 3517162 & $2007 / 2 / 7$ & 2008/10/14 & United States & Wm. Wrigley Jr. Company & Wrigleys \\
\hline 65 & 1633554 & $1990 / 3 / 12$ & $1991 / 1 / 29$ & France & Auchan & Auchan \\
\hline 66 & 3739986 & $2009 / 7 / 6$ & $2010 / 1 / 19$ & United States & $\begin{array}{l}\text { The Chase Manhattan } \\
\text { Corporation }\end{array}$ & Chase \\
\hline 67 & 1448362 & $1986 / 9 / 15$ & $1987 / 7 / 21$ & Japan & Nissan Group & Nissan \\
\hline 68 & 1721830 & $1991 / 4 / 24$ & $1992 / 10 / 6$ & United States & Dhl Corporation & DHL \\
\hline 69 & 3413407 & $2006 / 7 / 19$ & $2008 / 4 / 15$ & United States & Federal Express Corporation & Fedex \\
\hline 70 & 1297161 & $1983 / 9 / 12$ & $1984 / 9 / 18$ & United States & Homer Tlc, Inc. & Home Depot \\
\hline 71 & 2994515 & $2004 / 2 / 2$ & $2005 / 9 / 13$ & Germany & Mobile Tele Systems & MTS \\
\hline 72 & 2699023 & 2006/9/19 & $2007 / 10 / 30$ & Russian & $\begin{array}{l}\text { Beeline Mobile } \\
\text { Telecommunications }\end{array}$ & Beeline \\
\hline 73 & 78718666 & $2005 / 9 / 22$ & $2006 / 12 / 26$ & Japan & Canon Group & Canon \\
\hline 74 & 3414110 & $2007 / 3 / 26$ & $2008 / 4 / 22$ & United States & Aldi CORPORATION & Aldi \\
\hline 75 & 0635518 & $1955 / 8 / 26$ & $1956 / 10 / 9$ & $\begin{array}{l}\text { United } \\
\text { Kingdom }\end{array}$ & Avon Products, Inc. & Avon \\
\hline 76 & 4030529 & $2010 / 7 / 20$ & $2011 / 9 / 27$ & Spain & $\begin{array}{l}\text { Industria De Diseno Textil, } \\
\text { S.A.(Inditex, S.A.) }\end{array}$ & Zara \\
\hline 77 & 4074558 & 2009/9/16 & $2011 / 12 / 20$ & United States & O2 Holdings Limited & $\mathrm{O} 2$ \\
\hline 78 & 2667489 & 2011/11/16 & $2012 / 8 / 7$ & $\begin{array}{l}\text { United } \\
\text { Kingdom }\end{array}$ & Standard Chartered Plc & $\begin{array}{l}\text { Standard } \\
\text { Chartered }\end{array}$ \\
\hline 79 & 3197810 & $1995 / 8 / 10$ & $2007 / 1 / 16$ & Austria & $\begin{array}{l}\text { T.C. Pharmaceutical Industries } \\
\text { Co., Ltd. }\end{array}$ & Red Bull \\
\hline 80 & 3110886 & $2004 / 8 / 23$ & $2006 / 7 / 4$ & China & China Merchants Bank & $\begin{array}{l}\text { China } \\
\text { Merchants } \\
\text { Bank }\end{array}$ \\
\hline 81 & 2040691 & $1996 / 4 / 24$ & $1997 / 2 / 25$ & United States & Yahoo! Inc. & Yahoo \\
\hline 82 & 1364533 & $1984 / 8 / 9$ & $1985 / 10 / 8$ & France & Hermes-Gestion & Hermes \\
\hline 83 & 2651489 & $2001 / 6 / 27$ & $2002 / 11 / 19$ & United States & Jpmorgan Chase \& Co. & J.P.Morgan \\
\hline 84 & 3773373 & $2009 / 5 / 13$ & $2010 / 4 / 6$ & United States & $\begin{array}{l}\text { The Procter \& Gamble } \\
\text { Company }\end{array}$ & Ariel \\
\hline 85 & 3534633 & $2007 / 1 / 12$ & $2008 / 11 / 18$ & United States & $\begin{array}{l}\text { The Procter \& Gamble } \\
\text { Company }\end{array}$ & Tide \\
\hline 86 & 0876292 & $2011 / 11 / 7$ & $2012 / 5 / 22$ & United States & Guccio Gucci Soc. R. L. & Gucci \\
\hline 87 & 85434372 & $2011 / 9 / 28$ & $2012 / 7 / 31$ & United States & $\begin{array}{l}\text { Mastercard International } \\
\text { Incorporated }\end{array}$ & Mastercard \\
\hline 88 & 1975880 & $1994 / 10 / 12$ & $1996 / 5 / 28$ & United States & Goldman, Sachs \& Co. & $\begin{array}{l}\text { Goldman } \\
\text { Sachs }\end{array}$ \\
\hline 89 & 1943361 & $1993 / 7 / 26$ & $1995 / 12 / 26$ & United States & Starbucks Corporation & Starbucks \\
\hline 90 & 3855302 & $2006 / 12 / 13$ & $2010 / 10 / 5$ & $\begin{array}{l}\text { United } \\
\text { Kingdom }\end{array}$ & Barclays Bank Plc & Barclays \\
\hline 91 & 3373041 & $2006 / 7 / 3$ & $2008 / 1 / 22$ & United States & $\begin{array}{l}\text { State Farm Mutual Automobile } \\
\text { Insurance Company }\end{array}$ & State Farm \\
\hline 92 & 2729993 & $2001 / 3 / 5$ & $2003 / 6 / 24$ & United States & $\begin{array}{l}\text { Morgan Stanley Dean Witter \& } \\
\text { Co. }\end{array}$ & $\begin{array}{l}\text { Morgan } \\
\text { Stanley }\end{array}$ \\
\hline 93 & 74261048 & $1992 / 3 / 31$ & $1992 / 11 / 24$ & United States & $\begin{array}{l}\text { Internationale Nederlanden } \\
\text { Bank N.V. }\end{array}$ & Ing \\
\hline 94 & 2281415 & $1998 / 7 / 31$ & $1999 / 9 / 28$ & United States & $\begin{array}{l}\text { Kentucky Fried Chicken } \\
\text { International Holdings, Inc. }\end{array}$ & $\mathrm{Kfc}$ \\
\hline 95 & 1659330 & $1990 / 5 / 7$ & $1991 / 10 / 8$ & Netherl & Inter Ikea Systems B.V. & Ikea \\
\hline 96 & 0936721 & $2007 / 7 / 6$ & 2008/7/1 & Germany & Beiersdorf Ag & Nivea \\
\hline 97 & 1401275 & $1980 / 3 / 24$ & $1986 / 7 / 15$ & United States & Esprit International & Esprit \\
\hline 98 & 2243427 & $1997 / 4 / 17$ & $1999 / 5 / 4$ & Brazil & Bradesco Bank & Bradesco \\
\hline 99 & 3130133 & $2004 / 11 / 16$ & $2006 / 8 / 15$ & Italy & Telecom Italia S.P.A. & Tim \\
\hline 100 & 4001201 & $2010 / 7 / 9$ & $2011 / 7 / 26$ & United States & Lf Corporation & Lowes \\
\hline
\end{tabular}




\subsection{Analyzing the One-Time Data}

Famous enterprises still own its trademarks legally and continuously, indicating that enterprises still use brand as their weapon. In addition, within the best 100 brands (as shown in Figure 2), almost half of the brand nationalities are America, following by German (9\%), England (7\%), Japan (6\%)..., etc. The ratio shows that advanced countries still hold the edge.

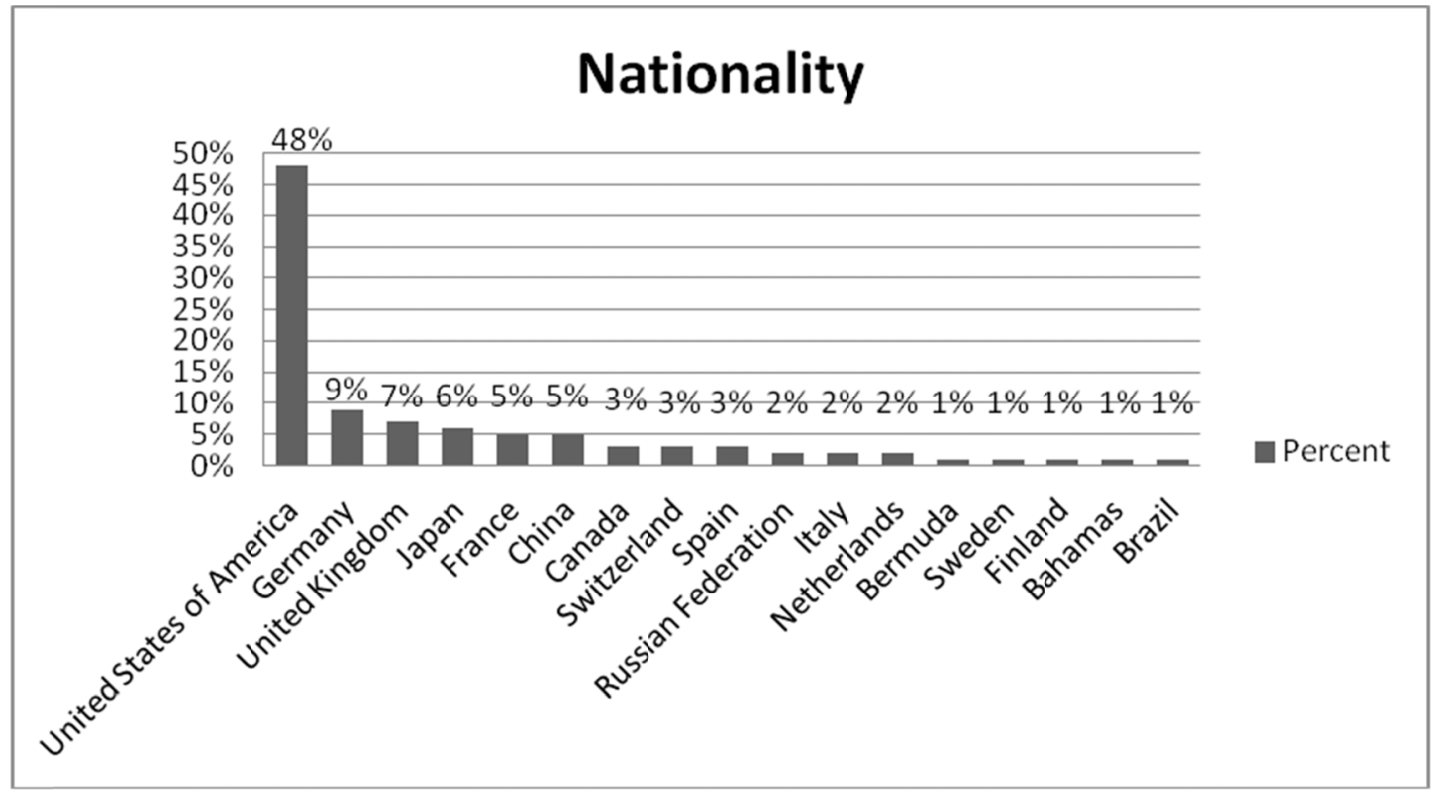

Figure 2. The statistics about the best 100 brands, published by Brandz in 2009

Due to the property of the best 100 brands belonging to different categories, the classification published by Interbrand and Brandz was taken as the standard to group the best 100 brands in 13 property categories, Table 3 .

Table 3 . The property categories of the one hundred brands

\begin{tabular}{|c|c|c|}
\hline No & Sector & Brand \\
\hline 1 & Technology & $\begin{array}{l}\text { Google, Microsoft, IBM, Apple, GE, Nokia, Blackberry, HP, SAP, Intel, Oracle, Cisco, } \\
\text { Dell, Accenture, Siemens, Canon, and Yahoo. }\end{array}$ \\
\hline 2 & Soft drinks & Cocacola, Pepsi, and Red Bull. \\
\hline 3 & Fast food & Mcdonals, Subway, Starbucks, and KFC. \\
\hline 4 & Mobile operators & $\begin{array}{l}\text { China Mobile, Vodafone, At\&T, Verizon, Docomo, Orange, Movistar, T Mobile, MTS, } \\
\text { Beeline, O2, and TIM. }\end{array}$ \\
\hline 5 & Retail & $\begin{array}{l}\text { Walmart, TESCO, Amazon, Carrefour, Ebay, Target, Auchan, Home Depot, ALDI, } \\
\text { IKEA, and Lowes. }\end{array}$ \\
\hline 6 & $\begin{array}{l}\text { Financial } \\
\text { Institutions }\end{array}$ & $\begin{array}{l}\text { ICBC, China Construction Bank, Bank Of China, HSBC, VISA, Wells Fargo, Santander, } \\
\text { Bank Of America, American Express, RBC, Citi, BBVA, TD, Chase, Standard } \\
\text { Chartered, China Merchants Bank, J.P.Morgan, Mastercard, Goldman Sachs, Barclays, } \\
\text { Morgan Stanley, and Bradesco. }\end{array}$ \\
\hline 7 & Cars & Toyota, BMW, Porsche, Benz, Honda, and Nissan. \\
\hline 8 & Personal care & Gillette, Pampers, Loreal, Colgate, AVON, and NIVEA. \\
\hline 9 & Luxury & LV, Hermes, and Gucci. \\
\hline 10 & Gaming consoles & Nintendo. \\
\hline 11 & Beer & Budweiser. \\
\hline 12 & Apparel & H\&M, Nike, Zara, and Esprit. \\
\hline 13 & Insurance & State Farm, and ING. \\
\hline 14 & Others & Marlboro, UPS, Disney, Wrigleys, DHL, Fedex, Ariel, and Tide. \\
\hline
\end{tabular}




\subsection{Analyzing the Second-Time Data}

To study the closer distance between trademark samples, the grouping results of the 86 trademark samples were organized into a diverse matrix (as shown in Appendix 1). In addition, the distance between trademark samples was analyzed by MDS and run on the SPSS software. As the result, the distribution of every trademark sample appeared in different dimensions.

Table 4 displays the stress coefficient and RSQ of one hundred brands in two to three dimensions. Higher dimension can give more ideal stress coefficient and RSQ. However, the main purpose of trademark map is to easily explain the similarity of trademarks. Due to the trademark distribution in 2D being easy to read, the distribution of trademark samples was shown in $2 \mathrm{D}$.

Table 4. The stress coefficient of one hundred brands in different dimensions

\begin{tabular}{lll}
\hline Dimension & Stress & RSQ \\
\hline 2 & 0.27200 & 0.66489 \\
3 & 0.17490 & 0.80484 \\
\hline
\end{tabular}

3.4 Building up the Trademark Map

According to the analyses of MDS, the 100 trademark samples judged by 86 subjects in $2 \mathrm{D}$ coordinate were displayed. Figure 3 shows the trademark map of one hundred brands aiming at normal consumers.

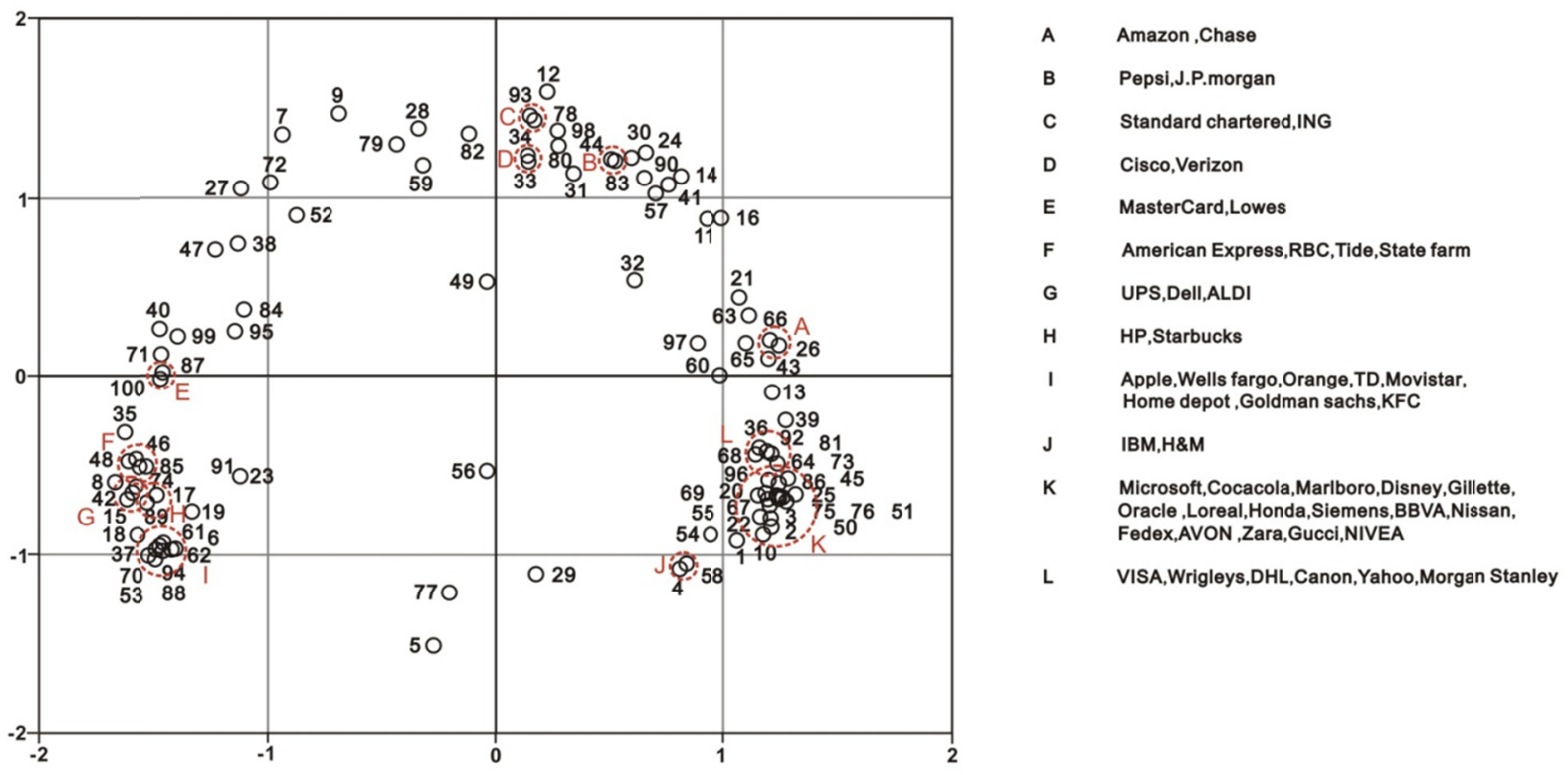

Figure 3. The trademark map of best 100 brands in the world

\subsection{Analyzing the Trademark Map}

With the comparison between Figure 1 and Figure 3, the distribution situation obviously separates from each quadrant. In first quadrant, there are mostly horizontal words combining with graphic trademarks, and the graphs appear on the right or left side of the words. The majority in the second quadrant are graphs above or beneath the words to combine graphic trademarks. There are mostly geometric graphs in the third quadrant, and word trademarks are placed in the forth quadrant. This design partition shows normal consumers judging trademark similarity by full appearance of the trademark (for example, outline and style) and using it for grouping trademarks.

Judging by the sparse or tight squeeze condition of trademark samples, close samples more likely lay in the third and forth quadrant, meaning the combination of words and graphs (in the second quadrant) can be identified more easier than geometric (in the third quadrant) and word trademarks (in the forth quadrant). Within group I and group K, there exist more overlapping situations like samples no.37, 70,61 and 53. This condition indicates that these samples have higher similarity and can cause confusion easily for consumers. 


\section{Discussion and Conclusion}

In the past, the brand strategy was usually planed subjectively by top managers of the enterprises. The concept of trademark map in this research can give feedback to top managers for more objective brand strategies and suppress their competitors effectively with unique brand. It is no doubt that trademark map will be one of the best tools for making brand strategies.

The five steps for building up trademark map are (1) deciding the range of trademark samples, (2) analyzing the first-time data, (3) analyzing the second-time data, (4) building up trademark map, and (5) analyzing trademark map. This standard procedure of trademark map building can assist enterprises in creating its own trademark maps with which enterprises can gain advantages of (1) knowing thoroughly about its own brand image, (2) being familiar with the oppression level of competitors, (3) monitoring the brand arrangement of competitors, and (4) planning the entire brand arrangement and investment clearly.

It is ambitious for creating an objective solution toward judging trademark similarity. The main goal is to provide references for brand design and protection by analyzing trademark data and building up trademark maps to display trademark similarity between enterprises. A trademark map can create strong effect on enterprises and brand designers. (1)For enterprises, they should build up trademark maps of their own and competitors' trademarks to understand the brand trademark arrangement from each other. Trademark maps can also be used for assisting enterprises in judging the identification and feasibility of trademark application, increasing distinguishability and avoiding tort. (2) For brand designers, their main job is trademark design that the sensitivity and opinion about brand symbols of designers quite differ from normal consumers. Therefore, designers are suggested to clarify its brand design and trademark design being highly distinguishable by applying trademark maps in the process of trademark design.

Every country has its own law to defense their trademarks as they are under the protection of owners' countries. However, the built trademark map is universal, which can be applied to different countries without any national limitations. Therefore, it is suggested that enterprises can build up trademark maps by following the five steps and develop them into a practical strategy of brand. The strategic tool can create a new edge for enterprises to face the knowledge economic era!

\section{References}

Arvidsson, A. (2006). Brand value. Journal of Brand Management, 13(3), 188-192. http://dx.doi.org/10.1057/palgrave.bm.2540261

Barnes, D. W. (2009). One Trademark Per Sourc. Tex Intellect Prop Law, 18(1), 1-54. http://dx.doi.org/10.2139/ssrn.1395014

Berry, L. L., \& Seltman, K. D. (2007). Building a strong services brand: Lessons from Mayo Clinic. Business Horizons, 50(3), 199-209. http://dx.doi.org/10.1016/j.bushor.2007.01.005

Berthon, P., Pitt, L. F., \& Campbell, C. (2009). Does brand meaning exist in similarity or singularity? Journal of Business Research, 62(3), 356-361. http://dx.doi.org/10.1016/j.jbusres.2008.05.015

BrandZ. (2009). Top 100 Most Valuable Global Brands Report. Retrieved from http://www.wpp.com/wpp/marketing/brandz/brandz-reports/

Delgado-Ballester, E., \& Munuera-Aleman, J. L. (2001). Brand trust in the context of consumer loyalty. European Journal of Marketing, 35(11/12), 1238-1258. http://dx.doi.org/10.1108/EUM0000000006475

Delgado-Ballester, E., \& Munuera-Aleman, J. L. (2005). Does brand trust matter to brand equity? Journal of Product \& Brand Management, 14(3), 187-196. http://dx.doi.org/10.1108/10610420510601058

Ewing, M.T. (2000). Brand and retailer loyalty: past behavior and future intentions. Journal of Product \& Brand Management, 9(2), 120-127. http://dx.doi.org/10.1108/10610420010322161

Gabay, G., Moskowitz, H. R., Beckley, J., \& Ashman, H. (2009). Consumer centered "brand value" of foods: drivers and segmentation. Journal of Product \& Brand Management, 18(1), 4-16. http://dx.doi.org/10.1108/10610420910933326

George, A. (2006). Brand rules: When branding lore meets trade mark law. Journal of Brand Management, 13(3), 215-232. http://dx.doi.org/10.1057/palgrave.bm.2540265

Huber, F., Vollhardt, K., Matthes, I., \& Vogel, J. (2009). Brand misconduct: Consequences on consumer-brand relationships. Journal of Business Research, 63(11), 1113-1120. http://dx.doi.org/10.1016/j.jbusres.2009.10.006 
Interbrand. (2010). Interbrand's Method for Valuating the Best Global Brands. Retrieved from http://www.interbrand.com/en/best-global-brands/previous-years/Best-Global-Brands-2010.aspx

Janssensa, F., Leta, J., Glanzel, W., \& Moor, B. D., (2006). Towards mapping library and information science. Information Processing and Management, 42(6), 1614-1642. http://dx.doi.org/10.1016/j.ipm.2006.03.025

Kort, P. M., Caulkins, J. P., Hartl, R. F., \& Feichtinger, G. (2006). Brand image and brand dilution in the fashion industry. Automatica, 42(8), 1363-1370. http://dx.doi.org/10.1016/j.automatica.2005.10.002

Mealema, Y., Yacobib,Y., \& Yaniv, G. (2010). Trademark infringement and optimal monitoring policy. Journal of Economics and Business, 62, 116-128. http://dx.doi.org/10.1016/j.jeconbus.2009.09.001

Olavarrieta, S., Torres, E., Vásquez-Parraga, A., \& Barra, C. (2009). Derived versus full name brand extensions. Journal of Business Research, 62(9), 899-905. http://dx.doi.org/10.1016/j.jbusres.2008.10.007

Palumbo, F., \& Herbig, P. (2000). The multicultural context of brand loyalty. European Journal of Innovation Management, 3(3), 116-124. http://dx.doi.org/10.1108/14601060010334876

Qi, H., Li, K., Shen, Y., \& Qu, W. (2010). An effective solution for trademark image retrieval by combining shape description and feature matching. Pattern Recognition, 43(6), 2017-2027. http://dx.doi.org/10.1016/j.patcog.2010.01.007

Rauyruen, P., Miller, K. E., \& Groth, M. (2009). B2B services: linking service loyalty and brand equity. Journal of Services Marketing, 23(3), 175-186. http://dx.doi.org/10.1108/08876040910955189

Shukla, P. (2009). Impact of contextual factors, brand loyalty and brand switching on purchase decisions. Journal of Consumer Marketing, 26(5), 348-357. http://dx.doi.org/10.1108/07363760910976600

Suzuki, D. Y. (2002). Trademark enforcement in the People's Republic of China. World Patent Information, 24(4), 293-295. http://dx.doi.org/10.1016/S0172-2190(02)00070-4

TIPO. (2012). Examination Guidelines on Likelihood of Confusion. Taiwan Intellectual Property Office. Retrieved from http://www.tipo.gov.tw/lp.asp?ctNode $=6822 \&$ CtUnit=3316\&BaseDSD $=7 \& \mathrm{mp}=2$

Torres-Moraga, E., Va'squez-Parraga, A. Z., \& Zamora-Gonza'lez, J. (2008). Customer satisfaction and loyalty: start with the product, culminate with the brand. Journal of Consumer Marketing, 25(5), 302-313. http://dx.doi.org/10.1108/07363760810890534

Veloutsou, C., \& Moutinho, L. (2009). Brand relationships through brand reputation and brand tribalism. Journal of Business Research, 62(3), 314-322. http://dx.doi.org/10.1016/j.jbusres.2008.05.010 